\title{
Economic Outcomes in Patients with Chemotherapy- Naïve Metastatic Castration-Resistant Prostate Cancer Treated with Enzalutamide or Abiraterone Acetate Plus Prednisone
}

\author{
Krishnan Ramaswamy - Stanislav Lechpammer · Jack Mardekian • \\ Ahong Huang · Neil M. Schultz • Rickard Sandin • Li Wang • \\ Onur Baser · Daniel J. George
}

Received: December 20, 2019 / Published online: February 28, 2020

(C) The Author(s) 2020

\begin{abstract}
Introduction: Prostate cancer (PC) is the second leading cause of cancer death among US men and accounts for considerable healthcare expenditures. We evaluated economic outcomes in men with chemotherapy-naïve metastatic castration-resistant PC (mCRPC) treated with enzalutamide or abiraterone acetate plus prednisone (abiraterone).
\end{abstract}

Results of this study were presented in part at the American Urological Association's annual meeting; 3-6 May 2019; Chicago, IL.

Enhanced Digital Features To view enhanced digital features for this article go to https://doi.org/10.6084/ m9.figshare.11831154.

Electronic Supplementary Material The online version of this article (https://doi.org/10.1007/s12325020-01260-x) contains supplementary material, which is available to authorized users.

K. Ramaswamy $(\bowtie) \cdot J$. Mardekian

Pfizer Inc., New York, NY, USA

e-mail: krishnan.ramaswamy@pfizer.com

S. Lechpammer

Pfizer Inc., San Francisco, CA, USA

A. Huang · L. Wang

STATinMED Research, Plano, TX, USA

N. M. Schultz

Astellas Pharma, Inc., Northbrook, IL, USA
Methods: We performed a retrospective analysis on 3174 men (18 years or older) utilizing the Veterans Health Administration (VHA) database from 1 April 2014 to 31 March 2018. Men with mCRPC were included if they had at least one pharmacy claim for enzalutamide or abiraterone (first claim date $=$ index date) following surgical or medical castration, had no chemotherapy treatment within 12 months prior to the index date, and had continuous VHA enrollment for at least 12 months preand post-index date. Men were followed until death, disenrollment, or end of study and were 1:1 propensity score matched (PSM). All-cause and PC-related resource use and costs per patient per month (PPPM) in the 12 months post index were compared between matched cohorts.

Results: We identified 1229 men with mCRPC prescribed enzalutamide and 1945 prescribed abiraterone with mean ages of 74 and 73 years,

R. Sandin

Pfizer Inc., Sollentuna, Sweden

O. Baser

The University of Michigan, Ann Arbor, MI, USA

D. J. George

Duke Cancer Institute, Duke University School of Medicine, Durham, NC, USA

O. Baser

MEF University, Istanbul, Turkey 
respectively. After PSM, each cohort had 1160 patients. The enzalutamide cohort had fewer all-cause $(2.51$ vs $2.86 ; p<0.0001)$ and PC-related outpatient visits (0.86 vs 1.03 ; $p<0.0001)$, with corresponding lower all-cause (\$2588 vs $\$ 3115 ; p<0.0001)$ and PC-related (\$1356 vs $\$ 1775 ; p<0.0001$ ) PPPM outpatient costs compared with the abiraterone cohort. All-cause total costs (medical and pharmacy) PPPM ( $\$ 8085$ vs $\$ 9092 ; p=0.0002$ ) and PC-related total costs PPPM (\$6321 vs $\$ 7280$; $p<0.0001)$ were significantly lower in the enzalutamide cohort compared with the abiraterone cohort.

Conclusions: Enzalutamide-treated men with chemotherapy-naïve mCRPC had significantly lower resource utilization and healthcare costs compared with abiraterone-treated men.

\section{PLAIN LANGUAGE SUMMARY}

Prostate cancer (PC) is the second leading cause of death among men with cancer in the USA. Healthcare costs associated with PC, including hospitalizations, outpatient visits, and medications prescribed to treat adverse effects, depend on the severity of the disease and intensity of treatment, but are generally very high. Enzalutamide and abiraterone acetate with prednisone (abiraterone) are both approved treatments for men with PC that does not respond to treatments that reduce the male hormone testosterone, known as castration-resistant PC (CRPC). These drugs are associated with varying treatment duration and different adverse effects, and therefore could result in differences in the use of healthcare resources and overall cost of treatment. Here we evaluated the healthcare resource utilization (HCRU), which was calculated as the average number of healthcare encounters, including inpatient stays, outpatient visits, and pharmacy visits, and length of inpatient stays, and treatment costs associated with use of enzalutamide or abiraterone by men with metastatic CRPC (mCRPC), who had not received prior chemotherapy in the Veterans Health Administration. We found that men with chemotherapy-naïve mCRPC treated with enzalutamide used less healthcare resources and incurred lower total healthcare costs than men treated with abiraterone. On average, all-cause total healthcare costs were $\$ 1007$ per patient per month lower and PCrelated total healthcare costs were $\$ 959$ per patient per month lower for patients treated with enzalutamide than those treated with abiraterone. These results support the hypothesis that the long-term HCRU and costs of enzalutamide may be lower compared with abiraterone.

Keywords: Abiraterone acetate; Enzalutamide; Healthcare costs; Prostate cancer; Survival

\section{Key Summary Points}

Why carry out this study?

Prostate cancer treatment can incur significant costs due to hospitalizations, outpatient visits, and treatment medications including prescriptions to treat adverse events related to treatment.

Limited real-world data have been published evaluating the treatment costs of secondary antiandrogen therapies enzalutamide and abiraterone acetate with prednisone (abiraterone) in men with chemotherapy-naïve metastatic castration-resistant prostate cancer (mCRPC).

\section{What did the study ask?}

We evaluated economic outcomes in men with chemotherapy-naïve mCRPC treated with enzalutamide or abiraterone in the Veterans Health Administration (VHA) database to determine if the healthcare resource utilization (HCRU) and costs were different between enzalutamide- and abiraterone-treated patients. 


\section{What were the study outcomes/conclusions?}

Men with chemotherapy-naïve mCRPC treated with enzalutamide had less HCRU and incurred lower total healthcare costs than those treated with abiraterone.

\section{What has been learned from the study?}

This is the first study to report real-world data on the HCRU and costs for patients with chemotherapy-naïve mCRPC taking enzalutamide and abiraterone using the VHA database, which includes a large patient population that is distinct from patients enrolled in clinical trials or who are commercially insured.

These results support the hypothesis that the long-term HCRU and costs of enzalutamide may be lower compared with abiraterone.

\section{INTRODUCTION}

Prostate cancer (PC) is the most common malignancy and the second leading cause of cancer-related death among men in the USA. An estimated 174,650 new cases of PC will be diagnosed in the USA in 2019, with 31,620 deaths $[1,2]$. PC cost the Medicare system over a 3 -year period (2004 to 2007) about $\$ 1.2$ billion in 2013 US dollars [3]. The cost depends on the severity of disease and the intensity of treatment. Within 5 years of follow-up, approximately $10-20 \%$ of all patients with PC develop castration-resistant PC (CRPC) [4], defined as PC that progresses despite the use of androgen deprivation therapy by surgical or medical castration. The majority of these patients (84\%) demonstrate radiographic findings of metastatic CRPC (mCRPC), and at least 5\% will progress from nonmetastatic CRPC to mCRPC within 2 years [5]. Patients with mCRPC account for approximately $10 \%$ of all PC cases [6]. Currently there are no curative treatment options

available; therefore, the prognosis is poor, with an estimated overall survival of less than 3 years $[4,7,8]$.

Docetaxel was the standard of care to treat mCRPC until the introduction of two androgen pathway-targeted treatments, enzalutamide (a second-generation androgen receptor inhibitor) and abiraterone acetate (an irreversible CYP17A1 inhibitor) combined with prednisone (abiraterone). These two agents received US Food and Drug Administration approval for use in mCRPC after docetaxel in August 2012 and April 2011, respectively, and then in chemotherapynaïve mCRPC in September 2014 and December 2012, respectively [9]. Enzalutamide and abiraterone have delayed deterioration of healthrelated quality of life and increased survival for patients with chemotherapy-naïve mCRPC in clinical trial settings $[10,11]$.

Clinical trials are informative in assessing efficacy and safety of treatments, but it is important to evaluate their impact on patient outcomes as well as payers in the real-word setting. Furthermore, it is anticipated that treatments will differ in healthcare resource utilization (HCRU) and costs, e.g., due to disparate treatment duration and adverse event profiles [10-12]. Abiraterone is administered together with prednisone, a corticosteroid that is used to reduce treatment-related symptom burden. Corticosteroids have been shown to be associated with toxicities, such as adrenal suppression, diabetes, osteoporosis, myopathy, infection, hypertension, glaucoma, and cardiovascular disease [13-16], with increased cost from cumulative exposure [17]. However, outcomes like HCRU and costs are usually not collected within clinical trials, and when collected, would reflect outcomes of a generally younger population with fewer comorbidities that are closely monitored. Similar to efficacy and safety outcomes, HCRU and costs are therefore expected to be different in a clinical practice setting, highlighting the need for realworld evidence generation.

Very few real-world studies have assessed HCRU and costs of novel therapies in mCRPC in the USA. A recent study, using real-world data from patients with chemotherapy-naïve mCRPC in a commercially insured population, reported 
lower HCRU and PC-related inpatient and emergency department costs for enzalutamide compared with abiraterone [18]. Another recent real-world study described treatment patterns, healthcare costs, and survival in patients with mCRPC who were commercially insured or Medicare covered and showed that enzalutamide and abiraterone were the most common first-line treatments (more than 65\% in total); however, no direct cost comparisons between the treatments were reported [19].

The cost of treatments to noncommercially insured populations such as the Veterans Health Administration (VHA) has not been investigated. The VHA is the largest integrated healthcare system in the USA and represents a different patient population than most commercial claims databases or fee-for-service Medicare, with more racial minorities and more patients who reside in lower-income areas [20]. Treatment patterns have also been shown to differ in the VHA compared with commercially insured patients [20]. PC is the most common cancer diagnosis among male US veterans [21]. In 2014, the incidence of newly diagnosed PC was 12,000 , and the prevalence rate of survivorship was 200,000 [22].

This study was designed to compare HCRU and costs in patients with chemotherapy-naïve mCRPC treated with enzalutamide or abiraterone in the VHA database. Our study addresses important gaps in the literature and contributes additional real-world evidence on key outcomes in patients with mCRPC treated with secondary antiandrogen therapy in the firstline setting. The study also assessed survival differences between enzalutamide and abiraterone [23], which will be reported in a separate paper.

\section{METHODS}

\section{Data Source}

In this retrospective observational study, enrollment, inpatient, outpatient, and pharmacy data were captured from 1 April 2013 to 31 March 2018 in the VHA database. Medical claims included diagnosis and procedure codes from the International Classification of
Diseases, Ninth and Tenth Revisions, Clinical Modification (ICD-9-CM and ICD-10-CM), Current Procedural Terminology, Version 4 procedure codes, and Healthcare Common Procedure Coding System codes. Outpatient pharmacy claims included National Drug Codes for dispensed medications. This study was exempt from institutional review board assessment because there was no extraction or use of any personally identifiable information.

\section{Patient Identification}

Eligible patients (18 years or older) had at least one medical claim with a diagnosis code for PC (ICD-9-CM: 185, ICD-10-CM: C61), evidence of surgical or medical castration, and a prescription claim for abiraterone or enzalutamide after castration at any time during the study. The first claim for enzalutamide or abiraterone between 1 April 2014 and 31 March 2018 was designated as the index date. Patients were required to have continuous VHA enrollment for at least 12 months pre- (baseline period) and at least 12 months post-index date (follow-up period) and were followed until the earliest occurrence of either death, disenrollment, or end of study. To ensure patients were newly initiating treatment, those with prescription claims for abiraterone or enzalutamide or who had other types of cancers during the pre-index period were excluded from the study.

\section{Patient Characteristics}

Demographic characteristics (age and race) were examined on the index date and clinical characteristics, including modified Quan-Charlson comorbidity index (CCI) scores, individual comorbidities (urinary tract infection, impotence, breast disorders, hypertension, stroke, angina pectoris perforation, arrhythmia, congestive heart failure, hyperlipidemia, low-extremity arterial occlusive disease, type 2 diabetes, liver damage/abnormality, acute coronary syndrome/myocardial infarction), and prior prostate cancer treatments, were measured during the pre-index period. 


\section{HCRU and Costs}

All-cause and PC-related HCRU and costs were evaluated for both cohorts during a 12-month post-index period. HCRU and costs were considered PC-related if there was an observed ICD9/ICD-10 PC diagnosis in a primary or secondary position on the medical claim. All-cause and PC-related HCRU were calculated as the average number of healthcare encounters, categorized as inpatient stays, outpatient visits, and pharmacy visits. All-cause and PC-related costs were calculated as inpatient stay costs, outpatient visit costs, pharmacy visit costs, total medical costs (sum of inpatient and outpatient visit costs), and total costs (sum of medical and pharmacy visit costs) adjusted to 2018 US dollars using the medical care component of the Consumer Price Index [24].

\section{Statistical Analysis}

Demographic and baseline clinical characteristics and HCRU costs were compared between the enzalutamide and abiraterone cohorts using standard descriptive statistics. For continuous variables, $p$ values were obtained using Student's $t$ tests; for categorical variables, $p$ values were obtained using Chi-squared tests. HCRU and costs were measured on a per patient per month (PPPM) basis during the 12 months post-index period. A sensitivity analysis was also performed using the end of the study as the end date rather than the 12-month follow-up. An additional analysis also evaluated outcomes in patients after treatment discontinuation with enzalutamide or abiraterone to the end of the study.

\section{Propensity Score Matching}

To adjust for potential selection bias, the study cohorts were matched using the propensity score matching (PSM) method, a statistical regression approach to remove major confounding influences on outcome assessment. These influential factors may be from patients' clinical history or baseline characteristics, largely leading to patient selection bias. Patients in the enzalutamide and abiraterone cohorts were
1:1 matched using a greedy $5 \rightarrow 3$-digit match based on an allowable absolute difference between propensity scores of 0.01 . Propensity scores were calculated in a logistic regression model controlling for baseline age, race, Quan-CCI score, and comorbidities. Standardized mean differences (SMDs), calculated as the difference in mean of a baseline variable between the two cohorts divided by the standard deviation of the variable, were used to check for balance in adjusted characteristics [25]. We selected a threshold of 10 to assess balance, in accordance with the methodology of previous studies $[26,27]$. In addition, standardized differences are indicative of practical/clinical significance; for this purpose, SMDs greater than 10 are indicative of practically significant differences between the treatment cohorts.

All statistical analyses were performed using SAS 9.4 (SAS Institute Inc., Cary, NC, USA).

\section{RESULTS}

A total of 5685 patients with mCRPC were identified with a prescription of enzalutamide or abiraterone. After exclusion of patients diagnosed with other types of cancer pre-index and patients with evidence of chemotherapy pre-index, 3174 patients (55.8\%) were included, 1229 in the enzalutamide cohort and 1945 in the abiraterone cohort (Fig. 1).

\section{Baseline Characteristics}

Demographic and baseline clinical characteristics are listed in Table 1. On average, patients in the enzalutamide cohort were older than patients in the abiraterone cohort ( 74 years vs 73 years; $\quad p=0.0112$ ). Enzalutamide-treated patients were more likely to have comorbid conditions, including arrhythmia $(8.87 \%$ vs $5.60 \% ; p=0.0004)$, congestive heart failure $(9.85 \%$ vs $6.43 \% ; p=0.0005)$, and type 2 diabetes $(35.15 \%$ vs $29.56 \% ; p=0.0010)$.

After PSM, 1160 enzalutamide-treated patients were matched 1:1 with abirateronetreated patients; both cohorts were well balanced on baseline characteristics (Table 1). 


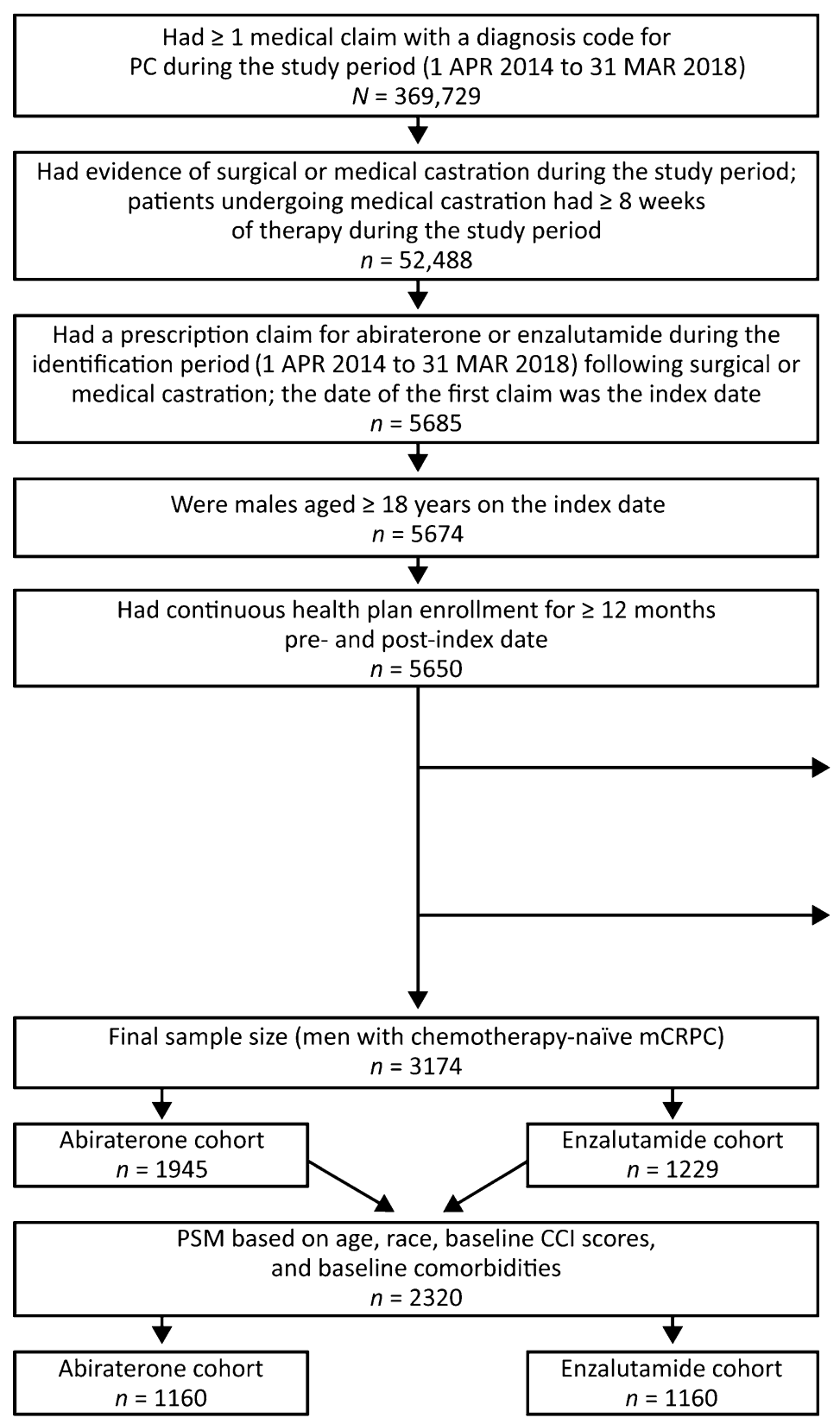

Excluded patients with presence of other cancers during the 12-month pre-index period $n=940$

Excluded patients with a prescription claim for abiraterone or enzalutamide or evidence of chemotherapy during the pre-index period $n=1536$

Fig. 1 Patient attrition. CCI Charlson comorbidity index, $P C$ prostate cancer, PSM propensity score matching

\section{Healthcare Resource Utilization}

All-cause and PC-related HCRU were mostly lower for enzalutamide-treated compared with abiraterone-treated patients during the 12-month post-index period (Fig. $2 \mathrm{a}, \mathrm{b}$ and Table 2). Enzalutamide-treated patients had significantly fewer all-cause outpatient visits PPPM (2.51 vs $2.86 ; p<0.0001$ ) and fewer all-cause pharmacy visits PPPM (2.95 vs 3.19; $p<0.0001)$. There were fewer PC-related inpatient stays PPPM (0.04 vs $0.05 ; p=0.0317)$, shorter inpatient length of stay (in days) PPPM (0.45 vs 0.63 ; $p=0.0291$ ), and fewer outpatient visits PPPM (0.86 vs $1.03 ; p<0.0001)$ in the enzalutamide cohort compared with the abiraterone cohort. For PC-related inpatient stays and length of stay, the SMDs were less than 10, indicating that the 


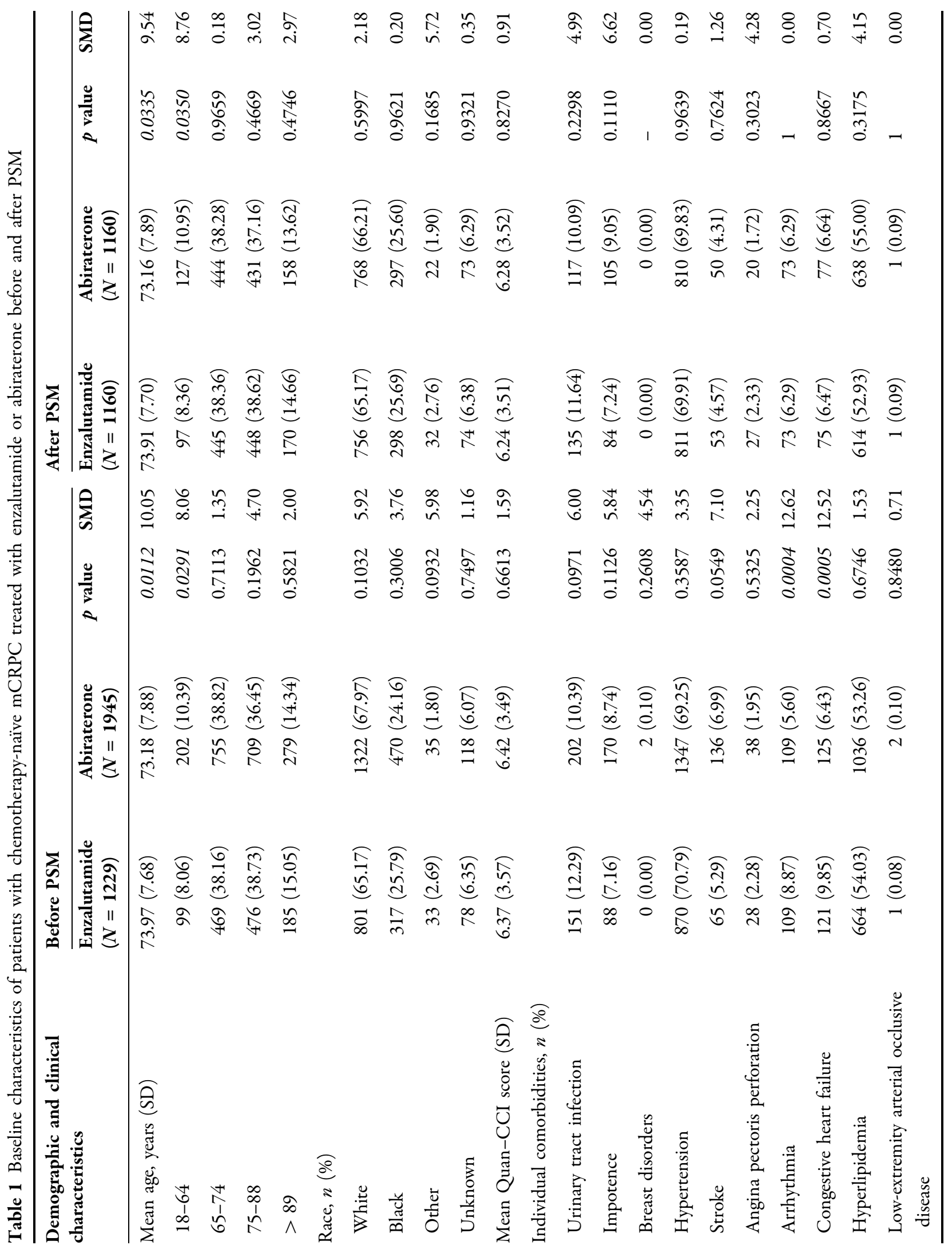




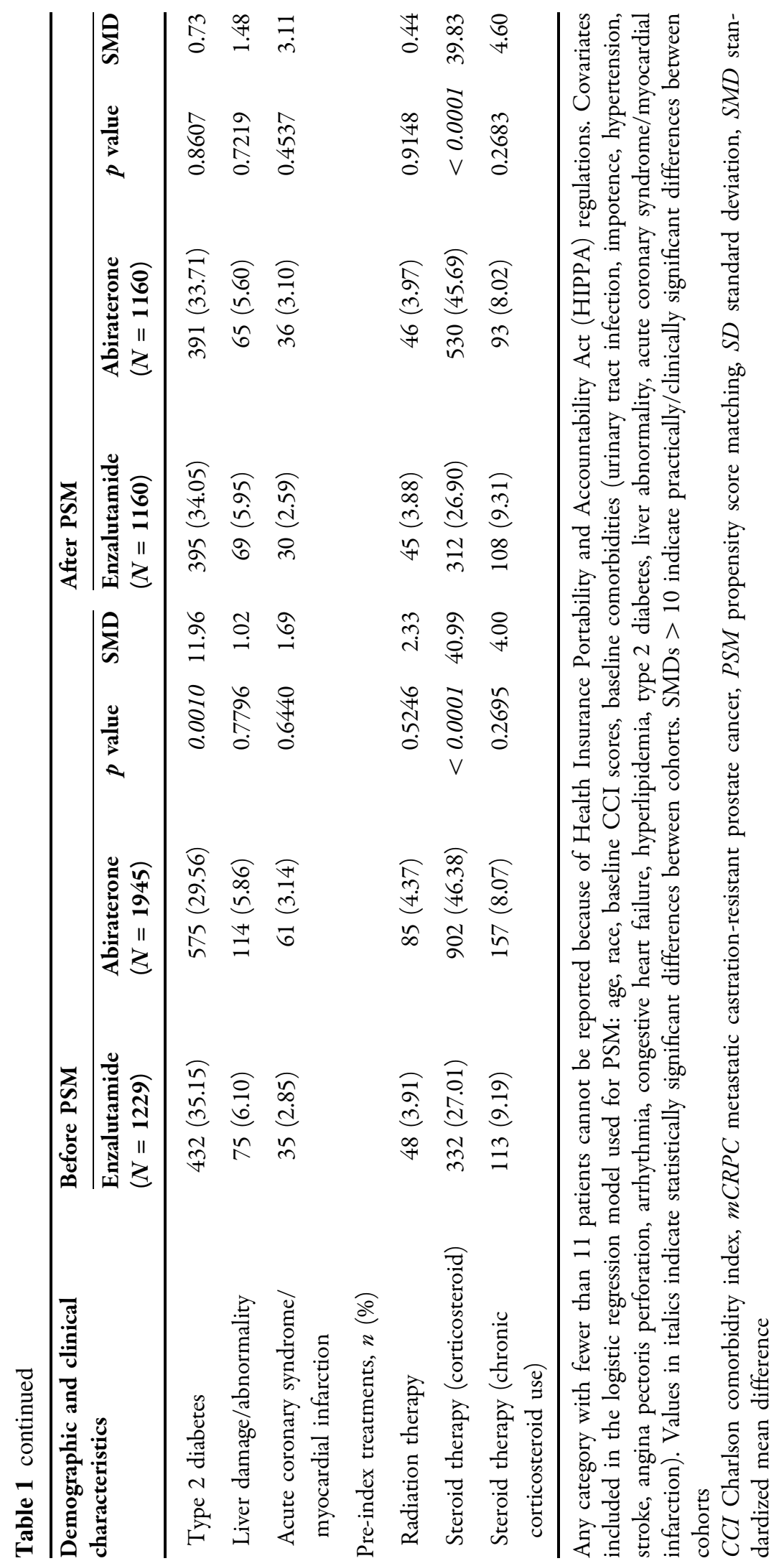



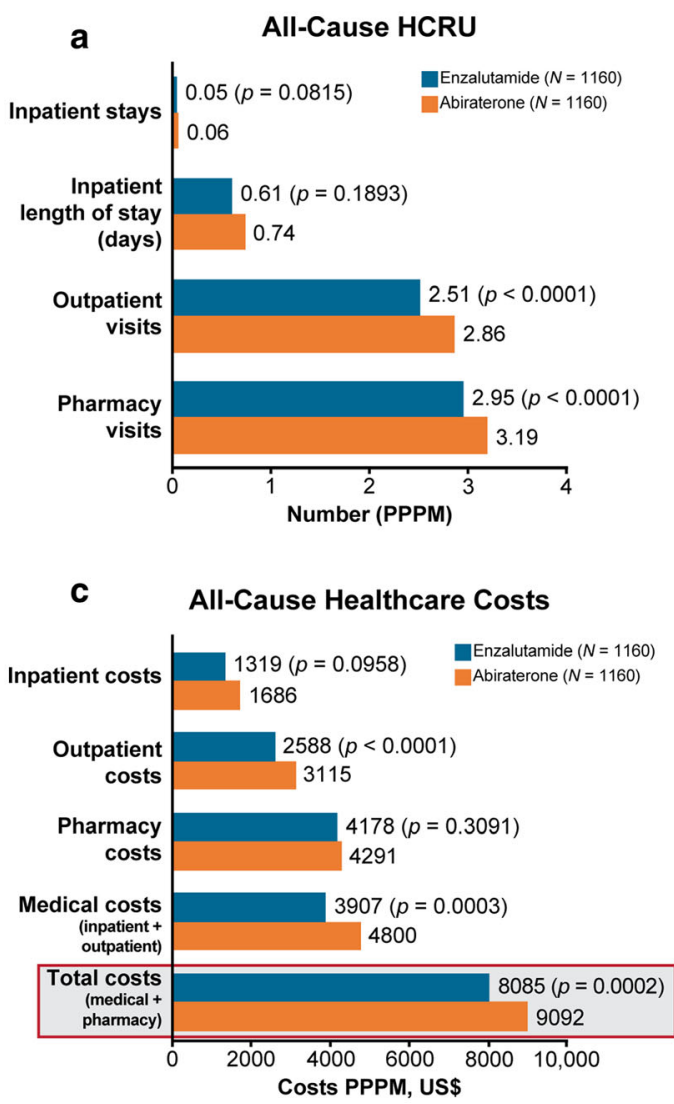

Fig. 2 All-cause (a) and PC-related (b) HCRU and allcause (c) and PC-related (d) costs among patients with chemotherapy-naive $\mathrm{mCRPC}$ treated with enzalutamide or abiraterone after PSM from index date through the

results were not practically significant. All-cause number of and inpatient length of stay (days) and PC-related number of pharmacy visits PPPM were similar between the cohorts.

\section{Healthcare Costs}

All-cause and PC-related healthcare costs were lower for enzalutamide-treated compared with abiraterone-treated patients 12 months post index (Fig. 2c, d and Table 2). Patients in the enzalutamide cohort incurred significantly lower all-cause outpatient costs PPPM (\$2588 vs $\$ 3115 ; p<0.0001)$, total medical costs PPPM (\$3907 vs $\$ 4800 ; p=0.0003$ ), and total costs PPPM ( $\$ 8085$ vs $\$ 9092 ; p=0.0002$ ). In both cohorts, PC-related costs accounted for a large proportion (ca. 80\%) of the all-cause costs. PCrelated inpatient costs PPPM (\$929 vs $\$ 1389$;
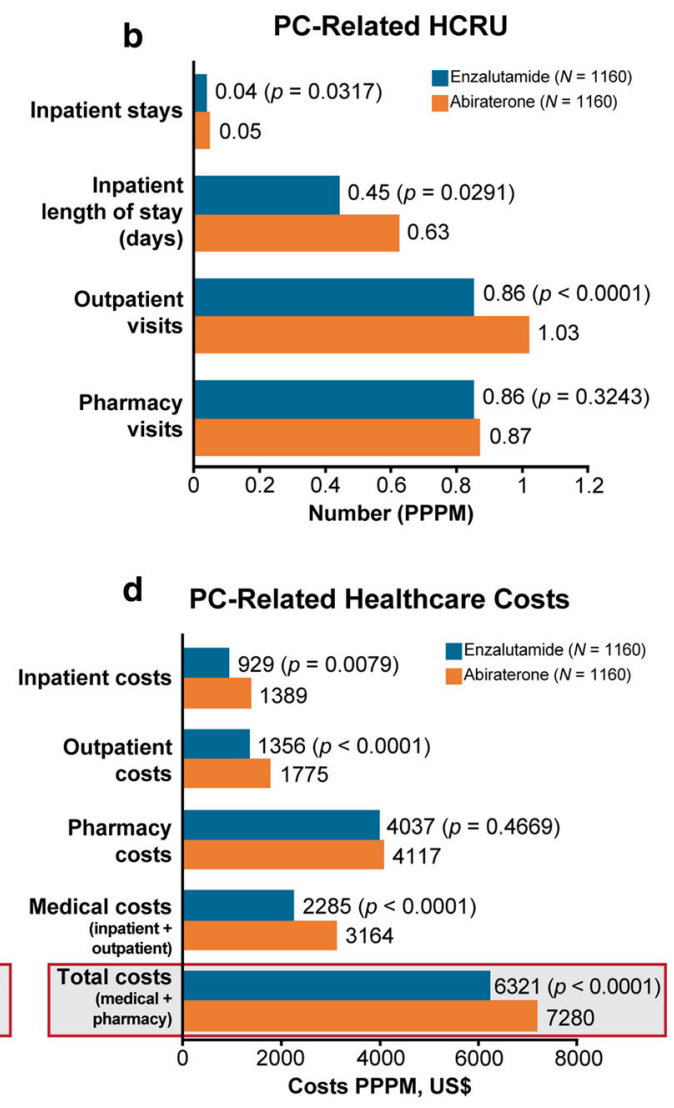

12-month follow-up period. $H C R U$ healthcare resource utilization, $m C R P C$ metastatic castration-resistant prostate cancer, $P C$ prostate cancer, $P P P M$ per patient per month, $P S M$ propensity score matching

$p=0.0079)$, outpatient visit costs PPPM (\$1356 vs $\$ 1775 ; p<0.0001)$, total medical costs PPPM (\$2285 vs $\$ 3164 ; p<0.0001)$, and total costs PPPM ( $\$ 6321$ vs $\$ 7280 ; p<0.0001)$ were significantly lower in the enzalutamide cohort compared with the abiraterone cohort. The proportion of patients who crossed over to the respective treatments (i.e., abiraterone followed by enzalutamide, 26\%; enzalutamide followed by abiraterone, $23 \%$ ) or followed first-line treatment with chemotherapy (abiraterone followed by chemotherapy, $9.2 \%$; enzalutamide followed by chemotherapy, 6.3\%) was similar.

\section{Sensitivity Analyses}

In a sensitivity analysis evaluating outcomes during the full study period, the median followup was 18.4 months (range 0.1-48.2) for the 
Table 2 Economic outcomes among patients with chemotherapy-naïve mCRPC treated with enzalutamide or abiraterone after PSM from the index date through the 12-month follow-up period

\begin{tabular}{|c|c|c|c|c|}
\hline 12-month follow-up outcomes & $\begin{array}{l}\text { Enzalutamide } \\
(N=1160)\end{array}$ & $\begin{array}{l}\text { Abiraterone } \\
(N=1160)\end{array}$ & $p$ value & SMD \\
\hline \multicolumn{5}{|l|}{ All-cause healthcare resource utilization } \\
\hline Any inpatient stay, $n(\%)$ & $291(25.09)$ & $334(28.79)$ & 0.0442 & 8.36 \\
\hline Any outpatient visit, $n$ (\%) & $1149(99.05)$ & $1157(99.74)$ & 0.0320 & 8.91 \\
\hline Any pharmacy visit, $n$ (\%) & $1160(100.00)$ & $1160(100.00)$ & NA & 0.00 \\
\hline Inpatient length of stay (in days), $\mathrm{PPPM} \pm \mathrm{SD}$ & $0.61 \pm 2.24$ & $0.74 \pm 2.43$ & 0.1893 & 5.45 \\
\hline Number of inpatient stays, $\mathrm{PPPM} \pm \mathrm{SD}$ & $0.05 \pm 0.13$ & $0.06 \pm 0.14$ & 0.0815 & 7.24 \\
\hline Number of outpatient visits, $\mathrm{PPPM} \pm \mathrm{SD}$ & $2.51 \pm 1.61$ & $2.86 \pm 1.69$ & $<0.0001$ & 20.94 \\
\hline Number of pharmacy visits, $\mathrm{PPPM} \pm \mathrm{SD}$ & $2.95 \pm 1.53$ & $3.19 \pm 1.48$ & $<0.0001$ & 16.32 \\
\hline \multicolumn{5}{|l|}{ PC-related healthcare resource utilization } \\
\hline Any inpatient stay, $n(\%)$ & $232(20.00)$ & $289(24.91)$ & 0.0046 & 11.79 \\
\hline Any outpatient visit, $n(\%)$ & $1072(92.41)$ & $1089(93.88)$ & 0.1624 & 5.80 \\
\hline Any pharmacy visit, $n(\%)$ & $1160(100.00)$ & $1160(100.00)$ & NA & 0.00 \\
\hline Inpatient length of stay (in days), $\mathrm{PPPM} \pm \mathrm{SD}$ & $0.45 \pm 1.68$ & $0.63 \pm 2.27$ & 0.0291 & 9.06 \\
\hline Number of inpatient stays, $\mathrm{PPPM} \pm \mathrm{SD}$ & $0.04 \pm 0.12$ & $0.05 \pm 0.13$ & 0.0317 & 8.93 \\
\hline Number of outpatient visits, $\mathrm{PPPM} \pm \mathrm{SD}$ & $0.86 \pm 0.72$ & $1.03 \pm 0.76$ & $<0.0001$ & 22.90 \\
\hline Number of pharmacy visits, $\mathrm{PPPM} \pm \mathrm{SD}$ & $0.86 \pm 0.53$ & $0.87 \pm 0.34$ & 0.3243 & 4.09 \\
\hline \multicolumn{5}{|l|}{ Mean all-cause healthcare costs, US\$ } \\
\hline Inpatient stay costs, $\mathrm{PPPM} \pm \mathrm{SD}$ & $1319 \pm 5030$ & $1686 \pm 5567$ & 0.0958 & 6.92 \\
\hline Outpatient visit costs, PPPM \pm SD & $2588 \pm 2238$ & $3115 \pm 2428$ & $<0.0001$ & 23.03 \\
\hline Pharmacy visit costs, $\mathrm{PPPM} \pm \mathrm{SD}$ & $4178 \pm 3161$ & $4291 \pm 2059$ & 0.3091 & 4.22 \\
\hline $\begin{array}{l}\text { Total medical (inpatient }+ \text { outpatient) costs, } \\
\mathrm{PPPM} \pm \mathrm{SD}\end{array}$ & $3907 \pm 5679$ & $4800 \pm 6284$ & 0.0003 & 14.92 \\
\hline Total (medical + pharmacy) costs, $\mathrm{PPPM} \pm \mathrm{SD}$ & $8085 \pm 6495$ & $9092 \pm 6676$ & 0.0002 & 15.29 \\
\hline \multicolumn{5}{|l|}{ Mean PC-related healthcare costs, US\$ } \\
\hline Inpatient stay costs, $\mathrm{PPPM} \pm \mathrm{SD}$ & $929 \pm 3314$ & $1389 \pm 4871$ & 0.0079 & 11.03 \\
\hline Outpatient visit costs, $\mathrm{PPPM} \pm \mathrm{SD}$ & $1356 \pm 1560$ & $1775 \pm 1910$ & $<0.0001$ & 24.03 \\
\hline Pharmacy visit costs, $\mathrm{PPPM} \pm \mathrm{SD}$ & $4037 \pm 3154$ & $4117 \pm 2044$ & 0.4669 & 3.02 \\
\hline $\begin{array}{l}\text { Total medical (inpatient }+ \text { outpatient) costs, } \\
\mathrm{PPPM} \pm \mathrm{SD}\end{array}$ & $2285 \pm 3870$ & $3164 \pm 5312$ & $<0.0001$ & 18.91 \\
\hline
\end{tabular}


Table 2 continued

\begin{tabular}{lllll}
\hline 12-month follow-up outcomes & $\begin{array}{l}\text { Enzalutamide } \\
(\boldsymbol{N}=\mathbf{1 1 6 0})\end{array}$ & $\begin{array}{l}\text { Abiraterone } \\
(\boldsymbol{N}=\mathbf{1 1 6 0})\end{array}$ & $\boldsymbol{p}$ value & SMD \\
\hline Total (medical + pharmacy) costs, PPPM $\pm \mathrm{SD}$ & $6321 \pm 5007$ & $7280 \pm 5708$ & $<0.0001$ & 17.87 \\
\hline
\end{tabular}

Values in italics indicate statistically significant differences between cohorts. SMDs $>10$ indicate practically/clinically significant differences between cohorts

$m C R P C$ metastatic castration-resistant prostate cancer, $P C$ prostate cancer, $N A$ not applicable, PPPM per patient per month, $P S M$ propensity score matching, $S D$ standard deviation, $S M D$ standardized mean difference

enzalutamide cohort and 19.1 months (range 0.4-48.7) for the abiraterone cohort. All-cause total costs PPPM were lower overall than in the 12-month follow-up period [mean all-cause costs were $\$ 8085$ vs $\$ 9092$ for the enzalutamide cohort vs the abiraterone cohort in the 12-month follow-up (Table 2) compared with $\$ 7643$ vs $\$ 8520$ in the full follow-up] (Table S1 in the supplementary material). The enzalutamide cohort had in general lower all-cause and PC-related HCRU and lower all-cause and PC-related costs than the abiraterone cohort (Table S1).

There were 667 patients in the enzalutamide cohort and 744 in the abiraterone cohort who discontinued treatment during the study period. There were significantly fewer all-cause outpatient visits PPPM in the enzalutamide cohort compared with the abiraterone cohort (2.61 vs 2.81 , respectively; $p=0.0381$ ). The remaining all-cause and PC-related HCRU outcomes were generally similar between the two cohorts (Table S2 in the supplementary material). Lower cost trends were observed for the enzalutamide cohort regarding all-cause and PC-related costs. However, there were significantly lower PC-related pharmacy ( $\$ 1252$ vs $\$ 1492 ; p=0.0033)$ and PC-related total costs PPPM ( $\$ 3755$ vs $\$ 4256 ; p=0.0496)$ in the enzalutamide cohort (Table S2).

\section{DISCUSSION}

This retrospective cohort study using recent real-world data from the VHA database provides evidence that enzalutamide-treated patients with chemotherapy-naïve mCRPC had lower HCRU and incurred lower healthcare costs than abiraterone-treated patients. Patient demographics and baseline characteristics were consistent with the demographic profile of patients enrolled in the VHA [28] and align with the mean ages reported in clinical studies of mCRPC [10, 11, 29, 30]. However, our study included a higher proportion of black patients compared with clinical trials $[10,11,29,30]$, reflecting the more diverse nature of the VHA database.

There were fewer all-cause outpatient and pharmacy visits for enzalutamide-treated patients compared with abiraterone-treated patients. Schultz et al. also found that treatment with enzalutamide, despite a higher occurrence of comorbid conditions, was associated with fewer inpatient and emergency department visits than abiraterone, likely attributed to corticosteroid use among abiraterone-treated patients [18]. In our PSM-matched cohorts, more abiraterone-treated patients used corticosteroids during the pre-index period than did enzalutamide-treated patients $(45.69 \%$ vs $26.90 \%$, respectively). Prednisone is prescribed concurrently with abiraterone to reduce occurrence of adverse events but is associated with immunosuppression, higher rate of infections, and more serious adverse events and comorbidities [7]. The difference in corticosteroid use pre-index could indicate that physicians chose to prescribe abiraterone to patients who were already taking corticosteroids for an unrelated comorbidity. Treatment of these corticosteroidsensitive comorbidities could require frequent outpatient or pharmacy visits for abirateronetreated patients and may explain why enzalutamide-treated patients had lower HCRU $[11,31]$. Cumulative use of corticosteroids 
before CRPC treatment initiation has also been shown to be associated with higher HCRU and costs [17].

Our study showed that enzalutamide-treated patients also had lower all-cause and PC-related healthcare costs. Massoudi et al. estimated that within a 1-year time horizon, the total cost per patient was $\$ 2666$ lower in the enzalutamide arm compared with the abiraterone arm in a model based on clinical trial data [32]. The cost difference in our study was much larger (a difference of approximately $\$ 12,000$ for all-cause and PC-related costs per year). Our results were based on real-world data including patients who may not be eligible for clinical trials, which may have led to more frequent HCRU and higher costs compared with a model based on a healthier patient population. Findings of lower all-cause emergency room and PC-related inpatient costs in enzalutamide-treated patients have also been previously reported, although these patients incurred higher all-cause and PCrelated pharmacy costs [18], whereas in our study, enzalutamide-treated patients had lower costs in all categories.

Enzalutamide and abiraterone have different toxicity profiles that will affect HCRU and costs differently. However, we present aggregate medical costs as accrued in the VA claims system and did not examine the specific cause of HCRUs and costs. There is limited information on toxicity-related costs with these agents. An administrative claims study, looking at mCRPC patients without differentiating between chemotherapy-naïve and post-chemotherapy patients, showed that there was a higher likelihood of having a broadly defined central nervous system (CNS) event with enzalutamide compared with abiraterone [33]. In a separate administrative claims study in a nonmetastatic prostate cancer population, CNS-related adverse events were associated with higher per patient per year HCRU with respect to inpatient and emergency department visits versus patients without CNS adverse events; however, no direct association with different drug treatment was reported as most patients were treated with bicalutamide $(n=477)$ and only a few patients on abiraterone or enzalutamide $(n=55)$ were included [34]. Other adverse event-related costs with second-generation androgen therapy, such as for cardiac disorder, infection, pneumonia, renal impairment, skeletal-related events, urinary tract infection, etc., have to our knowledge not been studied. Further research into the relative impact of adverse events of interest with the treatment of enzalutamide or abiraterone is warranted.

Given that about $75 \%$ of patients with mCRPC have overall survival longer than 12 months after initiation of abiraterone [35], we examined HCRU and costs until death or the end of study. All-cause and PC-related PPPM HCRU and costs overall were lower in the full follow-up period than in the first year of followup, mainly owing to lower pharmacy costs. This suggests that patients with mCRPC utilize more health services in the first year post-therapy initiation. Nonetheless, HCRU and costs were still found to be lower among enzalutamidetreated patients compared with abirateronetreated patients.

All-cause and PC-related HCRU and costs post discontinuation until the end of follow-up were also assessed among patients who discontinued. Results exhibited that all-cause and PCrelated HCRU and costs were also lower for enzalutamide-treated patients compared with abiraterone-treated patients. We did not assess what treatments were used post discontinuation, but the lower pharmacy costs are likely explained by use of low cost chemotherapy in later lines and limited PC drug treatment at the final stage of disease. Future studies should observe HCRU and costs pre- and post-switching among patients who experienced disease progression to reveal important information on the cost of disease progression.

\section{Limitations}

Findings of this study are subject to inherent limitations of administrative claims databases, such as coding errors or diagnoses entered for administrative processing rather than clinical completeness. Medications filled over-thecounter or provided as samples by the physician are not observed in the claims data. The presence of a diagnosis code on a medical claim 
does not necessarily indicate a positive presence of disease. The general applicability of our findings to younger patients requires further study. Although we adjust for differences in baseline patient characteristics to the extent possible, we cannot exclude that biases in prescribing patterns not revealed by these claims data could account for differences in prognosis, tolerance, and outcomes. We did not delineate what costs were attributable to the use of enzalutamide or abiraterone, for instance whether or not hospital visits were treatment related or unrelated to enzalutamide or abiraterone. Also, this analysis was completed at a time when abiraterone was still patent protected. Pricing of generic equivalents may alter cost analyses in future studies.

\section{CONCLUSIONS}

Among VHA patients with mCRPC, those treated with enzalutamide had less HCRU and incurred lower healthcare costs (on average \$1007 PPPM for all-cause and \$959 PPPM for PC-related total costs) than patients treated with abiraterone, supporting the hypothesis that the long-term costs and HCRU of enzalutamide may be lower compared with abiraterone. Additional studies within other healthcare settings are needed to provide a more complete picture of the resource use and costs in mCRPC patients treated with enzalutamide and abiraterone.

\section{ACKNOWLEDGEMENTS}

Funding. The study, Rapid Service Fee, and Open Access fee were funded by Pfizer Inc. (New York, NY, USA) and Astellas Pharma, Inc. (Northbrook, IL, USA). This study was sponsored by Pfizer Inc. (New York, NY, USA), and Astellas Pharma, Inc. (Northbrook, IL, USA), the co-developers of enzalutamide, and was conducted by STATinMED (Plano, TX, USA). STATinMED received compensation from the sponsors for the overall conduct of the study and development of this manuscript. All authors had full access to all of the data in this study and take complete responsibility for the integrity of the data and accuracy of the data analysis.

Medical Writing and Editorial Assistance. Medical writing and editorial assistance funded by Pfizer Inc. (New York, NY, USA) and Astellas Pharma, Inc. (Northbrook, IL, USA) were provided by Stephanie Vadasz, PhD, and Dena McWain of Ashfield Healthcare Communications (Middletown, CT, USA).

Authorship. All named authors meet the International Committee of Medical Journal Editors (ICMJE) criteria for authorship for this article, take responsibility for the integrity of the work as a whole, and have given their approval for this version to be published.

Disclosures. Krishnan Ramaswamy, Stanislav Lechpammer, Jack Mardekian, and Rickard Sandin are employees of Pfizer Inc. and own stock or stock options. Neil M. Schultz is an employee of Astellas Pharma Inc. and owns stock or stock options. Ahong Huang is an employee of and Li Wang is a former employee of STATinMED; STATinMED received compensation from the sponsors for the overall conduct of the study and development of this manuscript. Onur Baser and Daniel J. George have nothing to disclose.

Compliance with Ethics Guidelines. This study was exempt from institutional review board assessment because there was no extraction or use of any personally identifiable information.

Data Availability. The datasets generated during and/or analyzed during the current study are available from the corresponding author on reasonable request.

Open Access. This article is licensed under a Creative Commons Attribution-NonCommercial 4.0 International License, which permits any non-commercial use, sharing, adaptation, distribution and reproduction in any medium or format, as long as you give appropriate credit to the original author(s) and the source, provide 
a link to the Creative Commons licence, and indicate if changes were made. The images or other third-party material in this article are included in the article's Creative Commons licence, unless indicated otherwise in a credit line to the material. If material is not included in the article's Creative Commons licence and your intended use is not permitted by statutory regulation or exceeds the permitted use, you will need to obtain permission directly from the copyright holder. To view a copy of this licence, visit http://creativecommons.org/licenses/by-nc/ $4.0 /$.

\section{REFERENCES}

1. SEER. SEER Explorer. Surveillance, Epidemiology, and End Results Program; National Cancer Institute. 2019. https://seer.cancer.gov/explorer/appli cation.php?site $=66 \&$ data_type $=4 \&$ graph_type $=2 \&$ compareBy=time_interval\&hdn_sex=2\&chk_race $1=1 \& c h k$ age_range_1=1\&chk_stage_106=106\&chk _time_interval_1=1\&chk_time_interval_5=5\&advo pt_precision=1\&advopt_display=1\&advopt_show $\mathrm{ci}=$ on\&showDataFor=race_1_and_age_range_1_and _stage_106. Accessed Nov 14, 2019.

2. Siegel RL, Miller KD, Jemal A. Cancer statistics, 2019. CA Cancer J Clin. 2019;69:7-34.

3. Trogdon JG, Falchook AD, Basak R, Carpenter WR, Chen RC. Total Medicare costs associated with diagnosis and treatment of prostate cancer in elderly men. JAMA Oncol. 2019;5:60-6.

4. Kirby M, Hirst C, Crawford ED. Characterising the castration-resistant prostate cancer population: a systematic review. Int J Clin Pract. 2011;65: 1180-92.

5. Merseburger AS, Alcaraz A, von Klot CA. Androgen deprivation therapy as backbone therapy in the management of prostate cancer. Onco Targets Ther. 2016;9:7263-74.

6. Scher HI, Solo K, Valant J, Todd MB, Mehra M. Prevalence of prostate cancer clinical states and mortality in the United States: estimates using a dynamic progression model. PLoS One. 2015;10: e0139440.

7. De Santis M, Saad F. Practical guidance on the role of corticosteroids in the treatment of metastatic castration-resistant prostate cancer. Urology. 2016;96:156-64.
8. Huang $\mathrm{X}$, Chau $\mathrm{CH}$, Figg WD. Challenges to improved therapeutics for metastatic castrate resistant prostate cancer: from recent successes and failures. J Hematol Oncol. 2012;5:35.

9. Kapoor A, Wu C, Shayegan B, Rybak AP. Contemporary agents in the management of metastatic castration-resistant prostate cancer. Can Urol Assoc J. 2016;10:E414-23.

10. Beer TM, Armstrong AJ, Rathkopf DE, et al. Enzalutamide in metastatic prostate cancer before chemotherapy. N Engl J Med. 2014;371:424-33.

11. Ryan CJ, Smith MR, de Bono JS, et al. Abiraterone in metastatic prostate cancer without previous chemotherapy. N Engl J Med. 2013;368:138-48.

12. Shore ND, Saltzstein D, Sieber P, et al. Results of a real-world study of enzalutamide and abiraterone acetate with prednisone tolerability (REAAcT). Clin Genitourin Cancer. 2019;17:457-63.

13. Dorff TB, Crawford ED. Management and challenges of corticosteroid therapy in men with metastatic castrate-resistant prostate cancer. Ann Oncol. 2013;24:31-8.

14. Shah M, Chaudhari S, McLaughlin TP, et al. Cumulative burden of oral corticosteroid adverse effects and the economic implications of corticosteroid use in patients with systemic lupus erythematosus. Clin Ther. 2013;35:486-97.

15. Sarnes E, Crofford L, Watson M, Dennis G, Kan H, Bass D. Incidence and US costs of corticosteroidassociated adverse events: a systematic literature review. Clin Ther. 2011;33:1413-32.

16. Liu D, Ahmet A, Ward L, et al. A practical guide to the monitoring and management of the complications of systemic corticosteroid therapy. Allergy Asthma Clin Immunol. 2013;9:30.

17. Schultz NM, Penson DF, Wilson SD, et al. Health care resource utilization and costs associated with corticosteroid use in patients with castration-resistant prostate cancer: an administrative claims analysis. J Manag Care Spec Pharm. 2019;25: 889-97.

18. Schultz NM, Flanders SC, Wilson S, et al. Treatment duration, healthcare resource utilization, and costs among chemotherapy-naive patients with metastatic castration-resistant prostate cancer treated with enzalutamide or abiraterone acetate: a retrospective claims analysis. Adv Ther. 2018;35: 1639-55.

19. Wen L, Valderrama A, Costantino ME, Simmons S. Real-world treatment patterns in patients with 
castrate-resistant prostate cancer and bone metastases. Am Health Drug Benefits. 2019;12:142-9.

20. Nambudiri VE, Landrum MB, Lamont EB, et al. Understanding variation in primary prostate cancer treatment within the Veterans Health Administration. Urology. 2012;79:537-45.

21. Zullig LL, Sims KJ, McNeil R, et al. Cancer incidence among patients of the U.S. Veterans Affairs health care system: 2010 update. Mil Med. 2017;182: e1883-91.

22. Skolarus TA, Hawley ST. Prostate cancer survivorship care in the Veterans Health Administration. Fed Pract. 2014;31:10-7.

23. Ramaswamy K, Lechpammer S, Mardekian J, et al. Survival rates and economic outcomes in chemotherapy-naive metastatic castrate resistant prostate cancer patients treated with abiraterone acetate or enzalutamide. J Urol. 2019;201:e242-3.

24. Bureau of Labor Statistics. Consumer Price Index. 2018. http://data.bls.gov. Accessed Oct 29, 2019.

25. Austin PC. Using the standardized difference to compare the prevalence of a binary variable between two groups in observational research. Commun Stat Simul Comput. 2009;38:1228-34.

26. Roberts $\mathrm{MH}$, Dalal AA. Clinical and economic outcomes in an observational study of COPD maintenance therapies: multivariable regression versus propensity score matching. Int J Chron Obstruct Pulmon Dis. 2012;7:221-33.

27. Li X, Keshishian A, Hamilton M, et al. Apixaban 5 and $2.5 \mathrm{mg}$ twice-daily versus warfarin for stroke prevention in nonvalvular atrial fibrillation patients: comparative effectiveness and safety evaluated using a propensity-score-matched approach. PLoS One. 2018;13:e0191722.

28. Bagalman E. The number of veterans that use VA health care services: a fact sheet. Congressional
Research Service. 2014. https://fas.org/sgp/crs/misc/ R43579.pdf. Accessed Oct 29, 2019.

29. Scher HI, Fizazi K, Saad F, et al. Increased survival with enzalutamide in prostate cancer after chemotherapy. N Engl J Med. 2012;367:1187-97.

30. de Bono JS, Logothetis CJ, Molina A, et al. Abiraterone and increased survival in metastatic prostate cancer. N Engl J Med. 2011;364:1995-2005.

31. Bui CN, Wang L, Baser O. Resource utilization and use of life-extending therapies and corticosteroids in prostate cancer patients with corticosteroid-sensitive comorbidities. Curr Med Res Opin. 2014;30: 2355-64.

32. Massoudi M, Balk M, Yang H, et al. Number needed to treat and associated incremental costs of treatment with enzalutamide versus abiraterone acetate plus prednisone in chemotherapy-naive patients with metastatic castration-resistant prostate cancer. J Med Econ. 2017;20:121-8.

33. Pilon D, Behl AS, Ellis LA, Robitaille MN, Lefebvre P, Dawson NA. Assessment of real-world central nervous system events in patients with advanced prostate cancer using abiraterone acetate, bicalutamide, enzalutamide, or chemotherapy. Am Health Drug Benefits. 2017;10:143-53.

34. Shah A, Shah R, Kebede N, et al. Real-world incidence and burden of adverse events among nonmetastatic prostate cancer patients treated with secondary hormonal therapies following androgen deprivation therapy. J Med Econ. 2020. https://doi. org/10.1080/13696998.2019.1705313.

35. Boegemann M, Khaksar S, Bera G, et al. Abiraterone acetate plus prednisone for the management of metastatic castration-resistant prostate cancer (mCRPC) without prior use of chemotherapy: report from a large, international, real-world retrospective cohort study. BMC Cancer. 2019;19:60. 